\title{
Prophylactic Uterine Artery Embolization Assisted Cesarean Section for the Prevention of Intrapartum Hemorrhage in High-Risk Patients
}

\author{
Qun Li · Zheng-Qiang Yang • Wasif Mohammed • \\ Yao-Liang Feng · Hai-Bin Shi · Xin Zhou
}

Received: 13 November 2013/Accepted: 19 January 2014/Published online: 13 February 2014

(c) The Author(s) 2014. This article is published with open access at Springerlink.com

\begin{abstract}
Purpose To evaluate the safety and efficacy of prophylactic uterine artery embolization (UAE)-assisted cesarean section for the prevention of intrapartum hemorrhage.

Materials and Methods Twelve consecutive pregnant women (mean age 31 years; range 25-38) with uterine scarring and placenta previa and/or placenta accreta underwent UAE in conjunction with cesarean section to prevent intrapartum hemorrhage. For UAE, the left uterine artery was catheterized prophylactically under fluoroscopic guidance before the cesarean section incision was made. After the infant had been delivered, bilateral UAE was performed with the placenta still in situ. After successful bilateral UAE, the placenta was detached from the uterine wall.

Results Technical success was achieved in all 12 cases. Ten patients retained their uterus, and the other 2 underwent hysterectomy. The mean operative blood loss was $1,391 \mathrm{~mL}$ (range $600-3,600 \mathrm{~mL}$ ). The total mean fluoroscopy time and mean absorbed dose (air kerma) were $9 \mathrm{~min}$ $40 \mathrm{~s}$ (range $4 \mathrm{~min} 35 \mathrm{~s}-15 \mathrm{~min} 24 \mathrm{~s}$ ) and $91.79 \mathrm{mGy}$ (range 30.2-171), respectively. The average fetal fluoroscopy time was $1 \mathrm{~min} 42 \mathrm{~s}$ (range $41 \mathrm{~s}$ to $3 \mathrm{~min} 16 \mathrm{~s}$ ) with an average X-ray dose of $17.66 \mathrm{mGy}$ (range 6.04-23.90).
\end{abstract}

Q. Li · Z.-Q. Yang $(\bowtie) \cdot$ W. Mohammed · Y.-L. Feng

H.-B. Shi

Department of Interventional Radiology, First Affiliate Hospital

of Nanjing Medical University, 300 Guang Zhou Road,

Nanjing 210029, Jiangsu Province, China

e-mail: ntdoctoryang@hotmail.com

\section{Zhou}

Department of Obstetrics, First Affiliate Hospital of Nanjing Medical University, 300 Guang Zhou Road, Nanjing 210029, Jiangsu Province, China
Conclusion UAE-assisted cesarean section is safe and effective in the prevention of intrapartum hemorrhage in patients with uterine scarring and/or placental abnormalities.

Keywords Uterine artery embolization . Intrapartum hemorrhage - Abnormal placentation . Hysterectomy · Cesarean section

\section{Introduction}

Abnormal placentation, which broadly comprises placenta previa, accreta, increta, and percreta, is a leading cause of massive peripartum hemorrhage [1]. It frequently leads to emergency hysterectomies, surgical manipulation of organs adjacent to the uterus (ureter and bladder), respiratory distress syndrome, kidney failure, and even death [2, 3]. Blood loss in cesarean sections among patients with placental accreta can vary from 3,000 to $5,000 \mathrm{~mL}$ [4]. These conditions have been associated with significant maternal morbidity and mortality, and their occurrence has been reported to be increasing in recent decades $[1,5,6]$.

Recently prophylactic hypogastric artery occlusion has been introduced to decrease intraoperative blood loss in patients at high risk for peripartum hemorrhage $[4,7]$. The procedure consists of bilateral placement of embolizing materials and/or balloon catheters by way of the femoral arteries under fluoroscopic guidance. The literature offers evidence on the utility of preoperative placement of hypogastric artery catheters in decreasing blood loss and improving surgical outcomes [4, 7]. However, radiation exposure to the fetus and uterus during preoperative placement of balloon catheters or angiocatheters remains controversial. In addition, detailed data on fetal radiation 
Table 1 Summary of patient characteristics

GA gestational age

\begin{tabular}{lllll}
\hline $\begin{array}{l}\text { Patient } \\
\text { no./age(y) }\end{array}$ & $\begin{array}{l}\text { GA } \\
\text { (week }+ \text { day) }\end{array}$ & $\begin{array}{l}\text { No. of previous } \\
\text { curettages }\end{array}$ & $\begin{array}{l}\text { No. of previous } \\
\text { cesarean sections }\end{array}$ & MRI or US findings \\
\hline $1 / 30$ & 36 & 4 & 1 & Placenta accreta and placenta previa \\
$2 / 38$ & $35+5$ & 8 & 1 & Placenta percreta and placenta previa \\
$3 / 35$ & $35+2$ & 3 & 1 & Placenta previa \\
$4 / 31$ & $33+2$ & 3 & 1 & Placenta accreta and placenta previa \\
$5 / 33$ & $35+1$ & 3 & 1 & Placenta previa \\
$6 / 31$ & $35+2$ & 2 & 1 & Placenta accreta and placenta previa \\
$7 / 35$ & 33 & 8 & 1 & Placenta accreta and placenta previa \\
$8 / 33$ & $34+1$ & 3 & 1 & Placenta previa \\
$9 / 26$ & $35+5$ & 4 & 1 & Placenta accreta and placenta previa \\
$10 / 30$ & 35 & 7 & 1 & Placenta accreta and placenta previa \\
$11 / 27$ & $39+5$ & 2 & 1 & Placenta previa \\
$12 / 27$ & $31+2$ & 3 & 1 & Placenta accreta and placenta previa \\
Mean/31.3 & $35+2$ & 4 & 1 & - \\
\hline
\end{tabular}

exposure and maternal radiation exposure to the uterus have not been reported. Furthermore, transfer of the patient from the angiography suite to an operating room after prophylactic catheter placement will increase the complexity and risk of the procedure, particularly in unstable patients [8].

In this study, we investigated the safety and efficacy of uterine artery embolization (UAE) with preoperative catheterization in the unilateral uterine artery for assisted cesarean section for preventing intrapartum hemorrhage in patients with uterine scarring and/or placental abnormalities. The fetal and maternal radiation exposure doses were also recorded and analyzed.

\section{Materials and Methods}

Our Institutional Review Board approved this study. Between February 2012 and 2013, the patient cohort consisted of 12 parturients ranging in age from 25 to 38 years (mean 31) who had placental accreta and/or placenta previa on ultrasonic Doppler and/or magnetic resonance imaging during the prenatal period. All patients had a history of at least one previous cesarean section. Their average number of previous pregnancies was 3.6 (range 2 to 8 ). All individuals had a clinical diagnosis of a potential high risk for massive intrapartum hemorrhage because of the abnormal placenta; the average gestational duration was 36 weeks (range 31 to 39; Table 1).

Before surgery, all patients provided written informed consent and were brought to a regular operating room fitted with a moveable $\mathrm{C}$-arm equipped with digital subtraction angiography (DSA; Philips Medical Systems, Best, Netherlands) and a conventional operating table. After anesthetizing the patient with an epidural block, preoperative placement of temporary bilateral ureteral stents under cystoscopy and vesical catheter insertion were performed. In each patient, a $5 \mathrm{~F}$ sheath was placed in the right common femoral artery using Seldinger technique. Selective catheterization of the left uterine artery was performed with a 5F Roberts uterine curve (RUC) catheter (Cook, Bloomington, IN) under fluoroscopic guidance. The cumulative radiation dose and fluoroscopy time during the selective catheterization of the left uterine artery were recorded with the fetus in situ. The cumulative radiation dose absorbed per patient (air kerma) was recorded using the DSA system during fluoroscopy. Then a traditional cesarean delivery procedure was performed. After the infant had been delivered and the umbilical cord clamped, the obstetrician clipped any bleeding vessels using hemostatic forceps and packed the vagina and the uterus with the placenta still in situ (Fig. 1).

Under fluoroscopic guidance, the left uterine artery was embolized first, following which the right uterine artery was selectively catheterized and embolized with Gelform sponge pledgets (absorbable gelform sponge; Jinling pharmaceutical company, Nanjing, China) mixed with contrast medium. Both uterine arteries were embolized until there was adequate stasis, and the catheter and sheath were kept stationary in case further embolization was necessary. The cumulative radiation dose and fluoroscopy time during bilateral UAE were recorded, and the complete placenta was removed manually from the uterine wall using the traditional caesarean section technique. If bleeding continued even after bilateral UAE and led to an unstable blood pressure, emergency hysterectomy was performed.

After closing the uterine lumen and abdominal cavity and observing no vaginal bleeding, the angiocatheter was removed under fluoroscopic guidance. The puncture point of the right femoral artery was compressed for more than 

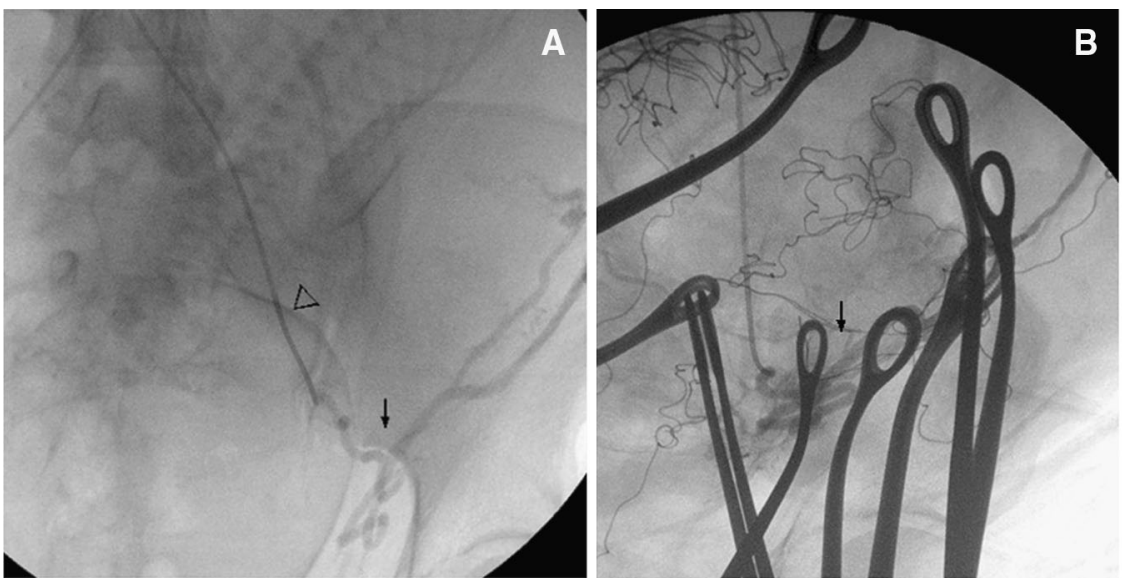

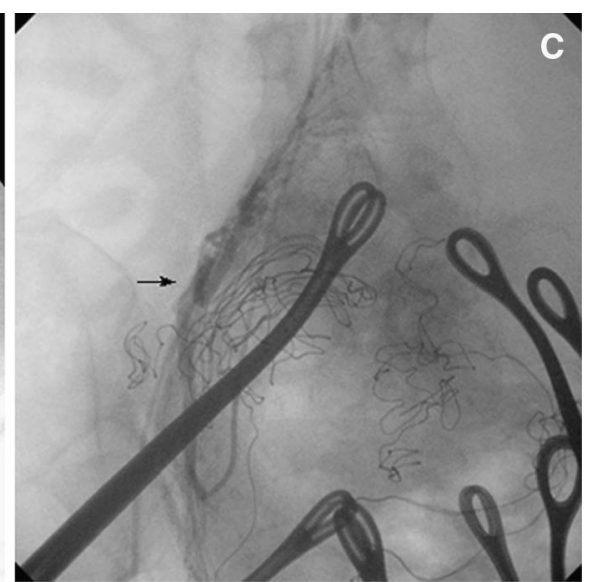

Fig. 1 Fluoroscopy images during procedure: Steps of prophylactic UAE-assisted cesarean section procedure in a 26-year-old patient (case no. 9). A A uterine catheter was inserted into the left uterine artery (arrow) superselectively before delivery. The fetus (arrowhead) is located within the uterus. B The left uterine artery (arrow) can be seen after successful embolization with ample gelfoam and contrast medium deposition. In addition, note the multiple shadows of hemostats used to stop bleeding after delivery. The placental outline can also be seen because it was still attached to the uterine wall. C The right uterine artery (arrow) can be seen after selective catheterization and embolization after infant delivery and before placental separation
$10 \mathrm{~min}$ for hemostasis. Intraoperative blood loss and volumes administered to the patient (crystalloid, colloid, and blood) were recorded. The length of surgery, infants' Apgar scores, and any complications after the procedure were recorded.

\section{Results}

No fetal or maternal mortality occurred in this group. Two parturients had refractory postpartum hemorrhage that was controlled by bilateral UAE followed by hysterectomy. The first of these patients had a large placenta previa that even covered the cervical os, and the second patient had placenta percreta, which extended into the uterine serosa and the wall of bladder. In 11 of 12 cases, the infant's Apgar score was 8 to 10 at 1 and $5 \mathrm{~min}$, respectively; the remaining neonate scored 5 at birth, but the score increased to 8 at 5 min after cardiopulmonary resuscitation. In 7 of 12 cases, the average fluoroscopy time used to place the RUC catheter was $1 \mathrm{~min} 42 \mathrm{~s}$ (range $41 \mathrm{~s}$ to $3 \mathrm{~min} 16 \mathrm{~s}$ ). The average X-ray dose was $17.66 \mathrm{mGy}$ (range 6.04-23.90), and the average dose area product was $0.493 \mathrm{mGym}^{2}$ (range 0.174-0.709). For the first five cases, we only recorded total fluoroscopy time and dose. The average total duration of fluoroscopy was $9 \mathrm{~min} 40 \mathrm{~s}$ (range $4 \mathrm{~min} 35 \mathrm{~s}$ to $15 \mathrm{~min} 24 \mathrm{~s}$ ). The average dose was $91.79 \mathrm{mGy}$ (range $30.2-71$ ), and the average dose area product was 2.37 $\mathrm{mGym}^{2}$ (range 0.849-4.09).

The average blood loss during the surgical procedure of all 12 cases was $1,391 \mathrm{~mL}$ (range 600-3,600), and the average urinary amount was $358 \mathrm{~mL}$ (range 200-500). The average volume of liquids administered was $2,333 \mathrm{~mL}$ (range 800-7,000). The average volume of colloid administered was $393 \mathrm{~mL}$ (range 200 to 910), and the average amount of blood transfused was 4.875 units (range 2 to 13 ) of packed red blood cells. The average duration of the surgical procedure was $2 \mathrm{~h} 19 \mathrm{~min}$ (range $1 \mathrm{~h} 20 \mathrm{~min}$ to $3 \mathrm{~h} 30 \mathrm{~min}$; Table 2). No further complications, such as infection and hemorrhage, were found in any of the cases. All patients had normal pulsation in the dorsal artery of the foot. The volume of vaginal bleeding was approximately $80 \mathrm{~mL}$ (range 20-150). The mean postoperative hospitalization time was 8.75 days (range 7-10). The documentation for every patient was complete.

\section{Discussion}

In this study, we showed that UAE-assisted cesarean section is an effective and safe method that is useful in the prevention of massive intrapartum hemorrhage in patients with abnormal placenta. In 10 of 12 patients, the uterus were successfully preserved with complete placental removal after prophylactic bilateral UAE.

Previous studies on conventional hysterectomy in similar patients found massive blood loss [9, 10]. Eller et al. identified 76 cases of patients with placenta accreta who underwent hysterectomy and reported that mean blood loss was $\sim 2.6 \mathrm{~L}$ [11]. Compared with those reports, there was much less blood loss (average 1,391 mL) in our study.

Weeks et al. [4] reviewed 21 patients undergoing hysterectomy who also underwent perioperative temporary balloon iliac artery occlusion. Although balloon occlusion 


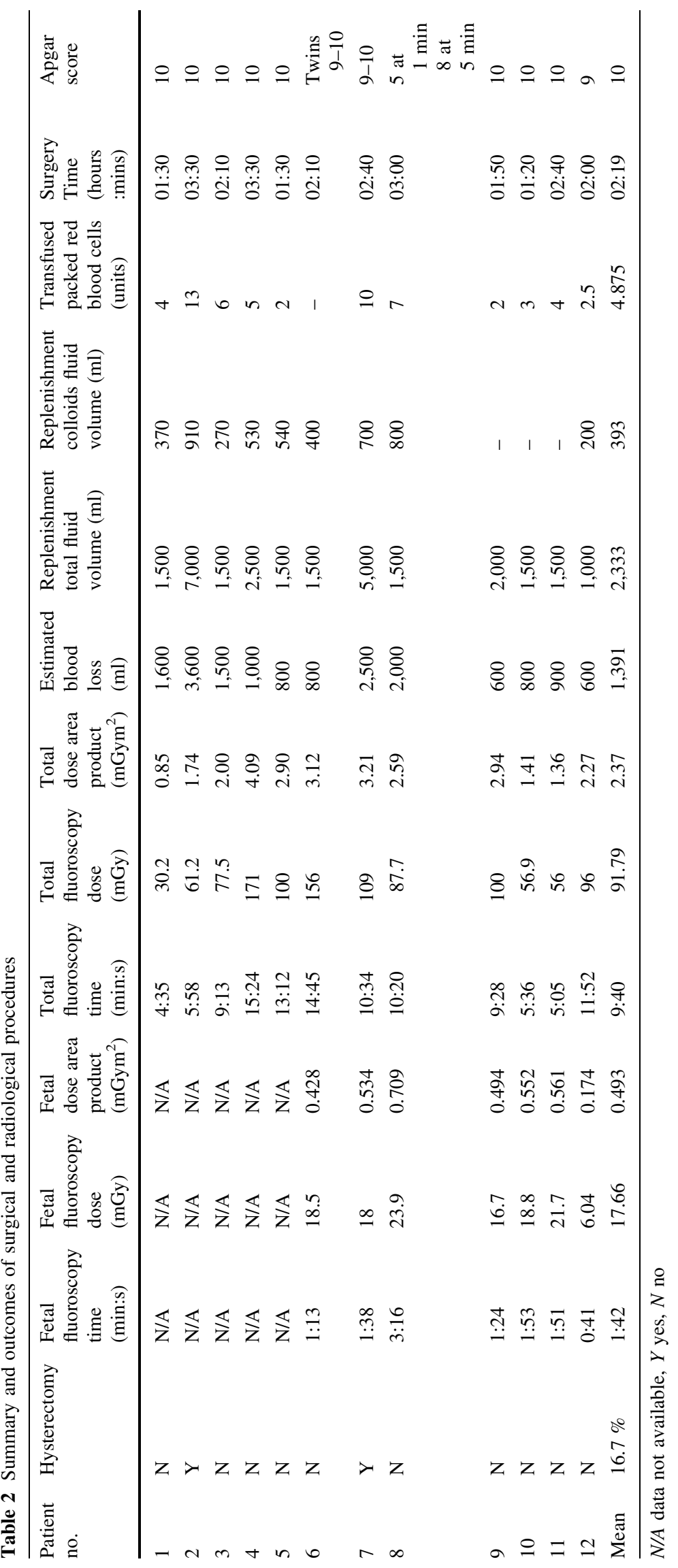


was performed before hysterectomy, there was still an estimated blood loss of $1,671.5 \mathrm{~mL}$. Another report employing similar methods reported estimated blood losses in successful cases ranging from 500 to $2,300 \mathrm{~mL}$ [8], which is similar to our study. However, in the same study, there were 6 cases $(54 \%)$ with the placenta left in situ, which might have led to a high incidence of recidivism. There was also massive blood loss in the two failed cases $(13,000$ and $10,000 \mathrm{~mL})$. The timing of insertion of the RUC catheter-after delivery of the baby in that study [8] compared with before delivery in our study - is the primary difference between the two reports. Delayed RUC insertion after delivery of the baby can lead to a delay in embolization, which could explain the higher volume of postpartum hemorrhage.

We found that fetal radiation exposure was minimal because only a single unilateral uterine artery was catheterized selectively under fluoroscopic guidance while the fetus was in utero. According to the National Council on Radiation Protection and Measurements, the risk to an infant from fluoroscopy dosage is increased significantly when the radiation exposure exceeds $150 \mathrm{mGy}[12,13]$. However, at doses of $<50 \mathrm{mGy}$, the risk of radiationinduced abnormality is considered negligible compared with baseline risks for all developmental abnormalities, e.g., $1 \%$ for mental retardation and $3 \%$ for spontaneous birth defects $[12,14]$. There was a wide range of total radiation dose and time because in some cases secondary UAE or other pelvic artery embolizations were performed when the obstetrician found further intraoperative hemorrhage during placental separation. It was convenient and flexible for us to perform repeat embolization because the DSA machine was stationed in the regular operating suite and the catheterization had been preserved.

Major complications associated with UAE in obstetric patients occur rarely with an overall complication rate of $\sim 9 \%$ [8]. These include transient fever, pelvic pain, transient foot ischemia, transient menelipsis, and abscess. In our study, all mothers had normal pulsation of the dorsal artery of the foot, and the average volume of vaginal bleeding was only $\sim 80 \mathrm{~mL}$. The mean hospitalization time was 8.75 days. Compared with a perioperative temporary occlusion study, which reported thrombogenesis in the femoral artery and other complications [4], our method seems to be much safer.

Another important innovation of our study was the timing of RUC insertion into the uterine artery. Some previous investigators did not use fluoroscopy before fetal delivery. Instead, they inserted a $5 \mathrm{~F}$ femoral arterial sheath $[8,15]$ before delivery to minimize fetal radiation. However, the main causes of UAE failure in these studies were vascular spasm with shock resulting from massive blood loss along with uterine artery movements during delivery, which made it difficult to accurately place the RUC into the uterine artery. Although our method of placing the RUC before delivery is simple and safe, we still must consider the decreased blood flow associated with fetal distress and the increased fluoroscopy exposure time if the RUC is placed in both uterine arteries. Hence, we elected to catheterize one unilateral uterine artery only before delivery to decrease the exposure time, and the Apgar score of all but one of the delivered fetuses was within the normal range.

A major limitation of our research was that it was retrospective in nature and the fetal fluoroscopy dose was only available for seven cases. Furthermore, this method must be validated by prospective controlled studies before mainstream implementation. Although we showed that there was minimal radiation exposure to the fetus during the positioning of the uterine artery RUC, which is below the specific threshold radiation level for teratogenesis, carcinogenesis is a stochastic effect of radiation, and there is no threshold level below which the risk of cancer can be eliminated completely [8, 12]. Hence, the safety of this method remains to be established by long-term studies.

In recent years, there has been significant interest in the potential role of interventional techniques in obstetrics, specifically in the control of peripartum hemorrhage [16]. Great attention has been paid to the potential of these procedures in improving maternal outcomes by decreasing morbidity and mortality. In our study, the blood loss volume was effectively controlled in most cases with an acceptable fluoroscopy dose. However, future randomized controlled trials and long-time follow-up studies are needed to determine whether the potential benefits of UAE during delivery outweigh the potential risks associated with fetal radiation exposure and vascular complications.

Conflict of interest No conflicts of interest exist for any of the authors involved in this manuscript.

Open Access This article is distributed under the terms of the Creative Commons Attribution License which permits any use, distribution, and reproduction in any medium, provided the original author(s) and the source are credited.

\section{References}

1. Bauer ST, Bonanno C (2009) Abnormal placentation. Semin Perinatol 33(2):88-96

2. Alexander JM, Wortman AC (2013) Intrapartum hemorrhage. Obstet Gynecol Clin North Am 40(1):15-26

3. Naz H, Sarwar I, Fawad A, Nisa AU (2008) Maternal morbidity and mortality due to primary $\mathrm{PPH}$ - experience at Ayub teaching hospital Abbottabad. J Ayub Med Coll Abbottabad 20(2):59-65

4. Weeks SM, Stroud TH, Sandhu J, Mauro MA, Jaques PF (2000) Temporary balloon occlusion of the internal iliac arteries for control of hemorrhage during cesarean hysterectomy in a patient 
with placenta previa and placenta increta. J Vasc Interv Radiol 11(5):622-624

5. Wu S, Kocherginsky M, Hibbard JU (2005) Abnormal placentation: twenty-year analysis. Am J Obstet Gynecol 192(5): 1458-1461

6. Silver RM, Landon MB, Rouse DJ, Leveno KJ, Spong CY, Thom EA et al (2006) Maternal morbidity associated with multiple repeat cesarean deliveries. Obstet Gynecol 107(6):1226-1232

7. Carnevale FC, Kondo MM, De Oliveira Sousa W Jr, Santos AB, da Motta Leal Filho JM et al (2011) Perioperative temporary occlusion of the internal iliac arteries as prophylaxis in cesarean section at risk of hemorrhage in placenta accreta. Cardiovasc Interv Radiol 34(4):758-764

8. Yu PC, Ou HY, Tsang LL, Kung FT, Hsu TY, Cheng YF (2009) Prophylactic intraoperative uterine artery embolization to control hemorrhage in abnormal placentation during late gestation. Fertil Steril 91(5):1951-1955

9. Guy GP, Peisner DB, Timor-Trisch IE (1990) Ultrasonographic evaluation of uteroplacental blood flow patterns of abnormally located and adherent placentas. Am J Obstet Gynecol 163(3):723-727

10. Tan SG, Jobling TW, Wallace EM, McNeilage LJ, Manolitsas T, Hodges RJ (2013) Surgical management of placenta accreta: a 10-year experience. Acta Obstet Gynecol Scand 92(4):445-450
11. Eller AG, Porter TF, Soisson P, Silver RM (2009) Optimal management strategies for placenta accreta. BJOG 116(5): 648-654

12. Patel SJ, Reede DL, Katz DS, Subramaniam R, Amorosa JK (2007) Imaging the pregnant patient for nonobstetric conditions: algorithms and radiation dose considerations. Radiographics 27(6): 1705-1722

13. Thabet A, Kalva SP, Liu B, Mueller PR, Lee SI (2012) Interventional radiology in pregnancy complications: indications, technique, and methods for minimizing radiation exposure. Radiographics 32(1):255-274

14. El-Khoury GY, Madsen MT, Blake ME, Yankowitz J (2003) A new pregnancy policy for a new era. AJR Am J Roentgenol 181(2):335-340

15. Diop AN, Chabrot P, Bertrand A et al (2010) Placenta accreta: management with uterine artery embolization in 17 cases. $\mathrm{J}$ Vasc Interv Radiol 21(5):644-648

16. Gonsalves M, Belli A (2010) The role of interventional radiology in obstetric hemorrhage. Cardiovasc Interv Radiol 33(5):887-889 